\title{
ESTIMATION FOR NONNEGATIVE LÉVY-DRIVEN ORNSTEIN-UHLENBECK PROCESSES
}

\author{
PETER J. BROCKWELL, ${ }^{* * *}$ Colorado State University \\ RICHARD A. DAVIS, ${ }^{* * *}$ Columbia University \\ YU YANG, ${ }^{*}$ Colorado State University
}

\begin{abstract}
Continuous-time autoregressive moving average (CARMA) processes with a nonnegative kernel and driven by a nondecreasing Lévy process constitute a very general class of stationary, nonnegative continuous-time processes. In financial econometrics a stationary Ornstein-Uhlenbeck (or CAR(1)) process, driven by a nondecreasing Lévy process, was introduced by Barndorff-Nielsen and Shephard (2001) as a model for stochastic volatility to allow for a wide variety of possible marginal distributions and the possibility of jumps. For such processes, we take advantage of the nonnegativity of the increments of the driving Lévy process to study the properties of a highly efficient estimation procedure for the parameters when observations are available of the CAR(1) process at uniformly spaced times $0, h, \ldots, N h$. We also show how to reconstruct the background driving Lévy process from a continuously observed realization of the process and use this result to estimate the increments of the Lévy process itself when $h$ is small. Asymptotic properties of the coefficient estimator are derived and the results illustrated using a simulated gammadriven Ornstein-Uhlenbeck process.
\end{abstract}

Keywords: Continuous-time autoregression; Ornstein-Uhlenbeck process; Lévy process; stochastic differential equation; sampled process

2000 Mathematics Subject Classification: Primary 62M10; 60H10

Secondary 62M09

\section{Introduction}

This paper is concerned with estimation of the parameters of a nonnegative Lévy-driven Ornstein-Uhlenbeck process and of the parameters of the background driving Lévy process, based on observations made at uniformly and closely-spaced times. We investigate the asymptotic properties of the estimator of the CAR(1) coefficient obtained by applying the method of Davis and McCormick (1989) to the estimation of the corresponding coefficient of the sampled AR(1) process. (The weak consistency of this estimator was shown by Jongbloed et al. (2005).) The estimator is then used to estimate the corresponding realization of the driving Lévy process. The exact recovery of the driving Lévy process requires continuous observation of the OrnsteinUhlenbeck process. The integral expressions determining the driving Lévy process are therefore replaced by approximating sums using the available discrete-time observations.

Received 5 March 2007; revision received 25 September 2007.

* Postal address: Department of Statistics, Colorado State University, Fort Collins, CO 80523-1877, USA.

** Email address: pjbrock@stat.colostate.edu

*** Postal address: Department of Statistics, Columbia University, New York, NY 10027, USA. 
In Section 2 we define the Lévy-driven CAR(1) process, $\{Y(t), t \geq 0\}$. In Section 3 we characterize the sampled AR(1) process, $\left\{Y_{n}^{(h)}=Y(n h), n=0,1,2, \ldots\right\}$, and the distribution of its driving white noise sequence in terms of the parameters of the underlying CAR(1) process and its driving Lévy process. The autoregressive coefficient of the sampled process is then estimated using the method of Davis and McCormick (1989). From the relation between the sampled and continuous-time processes, we then obtain corresponding parameter estimates for the CAR(1) process. The idea of using the sampled process to estimate the parameters of the underlying continuous-time process was first used by Phillips (1959), but in our case the nondecreasing property of the driving Lévy process and the nonnegativity of the corresponding discrete-time increments permits a very large efficiency gain. In Section 4 we show how to recover the driving Lévy process under the assumption that the process is observed continuously, and then approximate the results using closely-spaced discrete observations. In Section 5 we derive the asymptotic distribution of the coefficient estimator when the driving Lévy process is a gamma process, and illustrate with a simulated example the performance of the estimators of both the CAR(1) parameters and the driving Lévy process. When the continuously observed process is available, the autoregression coefficient can be identified with probability 1 . This is discussed in Section 6.

\section{Stationary Lévy-driven Ornstein-Uhlenbeck processes}

In order to define the stationary Lévy-driven Ornstein-Uhlenbeck (or CAR(1)) process, we first record a few essential facts concerning Lévy processes. (For a detailed account of integration with respect to Lévy processes, see Protter (2004).) Suppose that we are given a filtered probability space $\left(\Omega, \mathcal{F},\left(\mathcal{F}_{t}\right)_{0 \leq t \leq \infty}, \mathrm{P}\right)$, where $\mathcal{F}_{0}$ contains all the P-null sets of $\mathcal{F}$ and $\left(\mathcal{F}_{t}\right)$ is right-continuous.

Definition 2.1. (Lévy process.) We have $\{L(t), t \geq 0\}$ is an $\left(\mathcal{F}_{t}\right)$-adapted Lévy process if $L(t) \in \mathcal{F}_{t}$ for all $t \geq 0, L(0)=0$ almost surely (a.s.), $L(t)-L(s)$ is independent of $\mathcal{F}_{s}$, $0 \leq s<t<\infty, L(t)-L(s)$ has the same distribution as $L(t-s)$, and $L(t)$ is continuous in probability.

Every Lévy process has a unique modification which is càdlàg (right continuous with left limits) and which is also a Lévy process. We shall therefore assume that our Lévy process has these properties. For nondecreasing Lévy processes, the Laplace transform $\tilde{f}_{L(t)}(s):=\mathrm{E}\left(\mathrm{e}^{-s L(t)}\right)$ has the form

$$
\tilde{f}_{L(t)}(s)=\mathrm{e}^{-t \Phi(s)}, \quad \operatorname{Re}(s) \geq 0,
$$

where

$$
\Phi(s)=m+\int_{(0, \infty)}\left(1-\mathrm{e}^{-s x}\right) v(\mathrm{~d} x),
$$

with $m \geq 0$ and $v$ a measure on the Borel subsets of $(0, \infty)$ satisfying

$$
\int_{(0, \infty)} \frac{u}{1+u} v(\mathrm{~d} u)<\infty
$$

The measure $v$ is called the Lévy measure of the process $L$, and $m$ is called the drift. There exists a wealth of possible marginal distributions for $L(t)$, attainable by a suitable choice of $m$ and $v$; see, for example, Barndorff-Nielsen and Shephard (2001). For second-order Lévy processes, $\mathrm{E}(L(1))^{2}<\infty$, and there exist real constants $\mu$ and $\sigma$ such that

$$
\mathrm{E} L(t)=\mu t \quad \text { and } \quad \operatorname{var}(L(t))=\sigma^{2} t \quad \text { for } t \geq 0 .
$$


To avoid problems of parameter identifiability in the CAR(1) process defined below, we assume throughout that $L$ is scaled so that $\operatorname{var}(L(1))=1$. Then $\operatorname{var}(L(t))=t$ for $t \geq 0$, and we shall refer to the process $L$ as a standardized second-order Lévy process. Throughout this paper, we shall be concerned with CAR(1) (or stationary Ornstein-Uhlenbeck) processes driven by standardized second-order nondecreasing Lévy processes. The Lévy-driven CAR(1) process is defined as follows.

Definition 2.2. (Lévy-driven CAR(1) process.) A CAR(1) process driven by the Lévy process $\{L(t), t \geq 0\}$ with parameters $a \in \mathbb{R}$ and $\sigma>0$, is defined to be a strictly stationary solution to the stochastic differential equation

$$
\mathrm{d} Y(t)+a Y(t) \mathrm{d} t=\sigma \mathrm{d} L(t) .
$$

For the special case in which $\{L(t)\}$ is a Brownian motion, (2.1) is interpreted as an Itô equation with solution $\{Y(t), t \geq 0\}$ satisfying

$$
Y(t)=\mathrm{e}^{-a t} Y(0)+\sigma \int_{0}^{t} \mathrm{e}^{-a(t-u)} \mathrm{d} L(u),
$$

where the integral is defined as the $L^{2}$ limit of approximating Riemann-Stieltjes sums. For any second-order driving Lévy process $\{L(t)\}$, the process $\{Y(t)\}$ can be defined in the same way, and if $\{L(t)\}$ is nondecreasing (and hence of bounded variation on compact intervals) then $\{Y(t)\}$ can also be defined pathwise as a Riemann-Stieltjes integral by (2.2). We can also write

$$
Y(t)=\mathrm{e}^{-a(t-s)} Y(s)+\sigma \int_{s}^{t} \mathrm{e}^{-a(t-u)} \mathrm{d} L(u) \quad \text { for all } t>s \geq 0,
$$

showing, by independence of the increments of $\{L(t)\}$, that $\{Y(t)\}$ is Markov. (For a general theory of integration with respect to semimartingales, and in particular with respect to Lévy processes, see Protter (2004).) Proposition 2.1, below, gives necessary and sufficient conditions for stationarity of $\{Y(t)\}$. For a proof, see Brockwell and Marquardt (2005).

Proposition 2.1. If $Y(0)$ is independent of $\{L(t), t \geq 0\}$ and $\mathrm{E}\left(L(1)^{2}\right)<\infty$ then $Y(t)$ is strictly stationary if and only if $a>0$ and $Y(0)$ has the distribution of $\sigma \int_{0}^{\infty} \mathrm{e}^{-a u} \mathrm{~d} L(u)$.

Remark 2.1. By introducing a second Lévy process $\{M(t), 0 \leq t<\infty\}$, independent of $L$ and with the same distribution, we can extend $\{Y(t), t \geq 0\}$ to a process with index set $(-\infty, \infty)$. We define the following extension of $L$ :

$$
L^{*}(t)=L(t) \mathbf{1}_{[0, \infty)}(t)-M(-t-) \mathbf{1}_{(-\infty, 0]}(t), \quad-\infty<t<\infty .
$$

Then, provided that $a>0$, the process $\{Y(t)\}$ defined by

$$
Y(t)=\sigma \int_{-\infty}^{t} \mathrm{e}^{-a(t-u)} \mathrm{d} L^{*}(u)
$$

is a strictly stationary process satisfying (2.3) (with $L$ replaced by $L^{*}$ ) for all $t>s$ and $s \in(-\infty, \infty)$. Henceforth, we refer to $L^{*}$ as the background driving Lévy process and denote it by $L$ for simplicity. 
Remark 2.2. From (2.4) we have the relation

$$
Y(t)=\mathrm{e}^{-a(t-s)} Y(s)+\sigma \int_{s}^{t} \mathrm{e}^{-a(t-u)} \mathrm{d} L(u), \quad t \geq s>-\infty .
$$

Taking $s=0$ and using the pathwise interpretation of the integral in (2.5), we can also write

$$
Y(t)=\mathrm{e}^{-a t} Y(0)+\sigma L(t)-a \sigma \int_{0}^{t} \mathrm{e}^{-a(t-u)} L(u) \mathrm{d} u, \quad t \geq 0,
$$

where the last integral is a Riemann integral and the equality holds for all finite $t \geq 0$ with probability 1 .

\section{Parameter estimation via the sampled process}

Setting $t=n h$ and $s=(n-1) h$ in (2.5), we see at once that, for any $h>0$, the sampled process $\left\{Y_{n}^{(h)}, n=0,1,2, \ldots\right\}$ is the discrete-time $\operatorname{AR}(1)$ process satisfying

$$
Y_{n}^{(h)}=\phi Y_{n-1}^{(h)}+Z_{n}, \quad n=0,1,2, \ldots,
$$

where

$$
\phi=\mathrm{e}^{-a h}
$$

and

$$
Z_{n}=\sigma \int_{(n-1) h}^{n h} \mathrm{e}^{-a(n h-u)} \mathrm{d} L(u) .
$$

The noise sequence $\left\{Z_{n}\right\}$ is independent and identically distributed (i.i.d.) and positive since $L$ has stationary, independent, and positive increments.

If the process $\{Y(t), 0 \leq t \leq T\}$ is observed at times $0, h, 2 h, \ldots, N h$, where $N=\lfloor T / h\rfloor$ (here $\lfloor\cdot\rfloor$ denotes the integer part function), then, since the innovations $Z_{n}$ of the process $\left\{Y_{n}^{(h)}\right\}$ are nonnegative and $0<\phi<1$, we can use the highly efficient Davis-McCormick estimator for $\phi$, namely

$$
\hat{\phi}_{N}^{(h)}=\min _{1 \leq n \leq N} \frac{Y_{n}^{(h)}}{Y_{n-1}^{(h)}} .
$$

This estimator was proposed by Jongbloed et al. (2005), who showed the weak consistency of the estimator as $N$ tends to $\infty$ with $h$ fixed. To obtain the asymptotic distribution of $\hat{\phi}_{N}^{(h)}$, we shall suppose that the distribution function $F$ of $Z_{n}$ satisfies $F(0)=0$ and that $F$ is regularly varying at 0 with exponent $\alpha$, i.e. that there exists $\alpha>0$ such that

$$
\lim _{t \downarrow 0} \frac{F(t x)}{F(t)}=x^{\alpha} \quad \text { for all } x>0 .
$$

(These conditions are satisfied by the gamma-driven CAR(1) process, as we show in Section 5.) Under these conditions on $F$, the results of Davis and McCormick (1989) imply that $\hat{\phi}_{N}^{(h)} \rightarrow \phi$ a.s. as $N \rightarrow \infty$ with $h$ fixed and that

$$
\lim _{N \rightarrow \infty} \mathrm{P}\left[k_{N}^{-1}\left(\hat{\phi}_{N}^{(h)}-\phi\right) c_{\alpha} \leq x\right]=G_{\alpha}(x),
$$


where $k_{N}=F^{-1}\left(N^{-1}\right), c_{\alpha}=\left(\mathrm{E} Y_{1}^{(h) \alpha}\right)^{1 / \alpha}$, and $G_{\alpha}$ is the Weibull distribution function,

$$
G_{\alpha}(x)= \begin{cases}1-\exp \left\{-x^{\alpha}\right\} & \text { if } x \geq 0 \\ 0 & \text { if } x<0\end{cases}
$$

From the observations $\left\{Y_{n}^{(h)}, n=0,1 \ldots, N\right\}$ we thus obtain the estimator $\hat{\phi}_{N}^{(h)}$ and, from (3.2), the corresponding estimator,

$$
\hat{a}_{N}^{(h)}=-h^{-1} \log \hat{\phi}_{N}^{(h)},
$$

of the CAR(1) coefficient $a$. Provided that the distribution function $F$ of the noise terms $Z_{n}$ in the discrete-time sampled process satisfies the conditions indicated above, we can also determine the asymptotic distributions of this estimator. In particular, using a Taylor series approximation, we find that

$$
\lim _{N \rightarrow \infty} \mathrm{P}\left[(-h) \mathrm{e}^{-a h} c_{\alpha} k_{N}^{-1}\left(\hat{a}_{N}^{(h)}-a\right) \leq x\right]=G_{\alpha}(x),
$$

where $G_{\alpha}$ is given in (3.5). Since $\operatorname{var}\left(Y^{(h)}\right)=\sigma^{2} / 2 a$, we use the estimator

$$
\hat{\sigma}_{N}^{2}=\frac{2 \hat{a}_{N}^{(h)}}{N} \sum_{i=0}^{N}\left(Y_{i}^{(h)}-\bar{Y}_{N}^{(h)}\right)^{2}
$$

where $\bar{Y}_{N}^{(h)}=\sum_{i=0}^{N} Y_{I}^{(h)} /(N+1)$, to estimate $\sigma^{2}$.

\section{Estimating the Lévy increments}

So far, we have made no assumptions about the driving Lévy process except for nonnegativity and existence of $\mathrm{E}\left(L(1)^{2}\right)$. In order to suggest an appropriate parametric model for $L$ and to estimate the parameters, it is important to recover an approximation to $L$ from the observed data. If the $\mathrm{CAR}(1)$ process is continuously observed on $[0, T]$ then the integrated form of (2.1) immediately gives

$$
L(t)=\sigma^{-1}\left(Y(t)-Y(0)+a \int_{0}^{t} Y(s) \mathrm{d} s\right)
$$

From (4.1), the increment of the driving Lévy process on the interval $((n-1) h, n h]$ is given by

$$
\Delta L_{n}^{(h)}:=L(n h)-L((n-1) h)=\sigma^{-1}\left(Y(n h)-Y((n-1) h)+a \int_{(n-1) h}^{n h} Y(u) \mathrm{d} u\right) .
$$

Replacing the CAR(1) parameters by their estimators and the integral by a trapezoidal approximation, we obtain the estimated increments

$$
\Delta \hat{L}_{n}^{(h)}=\hat{\sigma}_{N}^{-1}\left(Y_{n}^{(h)}-Y_{n-1}^{(h)}+\frac{\hat{a}_{N}^{(h)} h\left(Y_{n}^{(h)}+Y_{n-1}^{(h)}\right)}{2}\right) .
$$

\section{The gamma-driven $\mathrm{CAR}(1)$ process}

In this section we illustrate the preceding estimating procedure for the case in which $L$ is a standardized gamma process. Thus, $L(t)$ has the gamma density $f_{L(t)}$ with exponent $\gamma t$, 
scale-parameter $\gamma^{-1 / 2}$, mean $\gamma^{1 / 2} t$, and variance $t$. The Laplace transform of $L(t)$ is

$$
\tilde{f}_{L(t)}(s):=\mathrm{Ee}^{-s L(t)}=\mathrm{e}^{-t \Phi(s)}, \quad \operatorname{Re}(s) \geq 0,
$$

where $\Phi(s)=\gamma \log (1+\beta s), \beta=\gamma^{-1 / 2}$, and $\gamma>0$.

Based on the $h$-spaced observations $\left\{Y_{n}^{(h)}, n=0,1, \ldots, N\right\}$, we estimate the discrete-time autoregression coefficient $\phi$ and the CAR(1) parameters $a$ and $\sigma^{2}$ using (3.3), (3.6), and (3.7), respectively. We then estimate the Lévy increments as in (4.2) and use them to estimate the parameter $\gamma$ of the standardized gamma process $L$. To obtain the asymptotic distributions of $\phi_{N}^{(h)}$ and $\hat{a}_{N}^{(h)}$ as $N$ tends to $\infty$ with $h$ fixed, we first show that the distribution function $F$ of $Z_{n}$ in (3.1) is regularly varying at 0 with exponent $\gamma h$, and then determine the coefficients $k_{N}=F^{-1}\left(N^{-1}\right)$ and $c_{\alpha}=\left(\mathrm{E} Y_{1}^{(h) \alpha}\right)^{1 / \alpha}$ in (3.4). To do so, we use the Laplace transform (5.1) to investigate the behavior of the density of $Z_{1}=\sigma \int_{0}^{h} \mathrm{e}^{-a(h-t)} \mathrm{d} L(t)$ near 0 .

Define $W_{h}:=Z_{1} / \sigma$. The Laplace transform of $W_{h}$ is

$$
\begin{aligned}
\tilde{f}_{W_{h}}(s) & =\exp \left(-\int_{0}^{h} \Phi\left(s \mathrm{e}^{-a t}\right) \mathrm{d} t\right) \\
& =\exp \left(-\int_{0}^{h} \gamma \log \left(1+\beta s \mathrm{e}^{-a t}\right) \mathrm{d} t\right) .
\end{aligned}
$$

The exponent in (5.2) has the power series expansion

$$
\begin{aligned}
-\int_{0}^{h} \gamma \log \left(1+\beta s \mathrm{e}^{-a t}\right) \mathrm{d} t= & -\gamma \int_{0}^{h} \log \left(s \beta \mathrm{e}^{-a t}\left(1+\frac{1}{\beta s \mathrm{e}^{-a t}}\right)\right) \mathrm{d} t \\
\approx & \log (s \beta)^{-\gamma h}+\frac{1}{2} \gamma a h^{2} \\
& +\gamma\left(\frac{1}{\beta s a}\left(1-\mathrm{e}^{a h}\right)-\frac{1}{4 \beta^{2} s^{2} a}\left(1-\mathrm{e}^{2 a h}\right)+\cdots\right)
\end{aligned}
$$

as $s \rightarrow \infty$. Hence, $\tilde{f}_{W_{h}}(s)$ has the corresponding expansion

$$
\tilde{f}_{W_{h}}(s) \approx \frac{\beta^{-\gamma h}}{s^{\gamma h}} \exp \left(\frac{1}{2} \gamma a h^{2}\right)+\frac{C_{1}}{s^{\gamma h+1}}+\frac{C_{2}}{s^{\gamma h+2}}+\cdots,
$$

where $C_{1}, C_{2}, \ldots$ are constants depending on $\gamma, \beta, h$, and $a$. Since $\tilde{f}_{Z_{1}}(s)=\tilde{f}_{W_{h}}(\sigma s)$,

$$
s^{\gamma h} \tilde{f}_{Z_{1}}(s) \rightarrow(\sigma \beta)^{-\gamma h} \exp \left(\frac{1}{2} \gamma a h^{2}\right) \quad \text { as } s \rightarrow \infty .
$$

By Theorem 30.2 of Doetsch (1974), the density $f_{Z_{1}}$ of $Z_{1}$ has the following expansion, in a neighborhood of 0 :

$$
f_{Z_{1}}(x)=\frac{(\sigma \beta)^{-\gamma h} x^{\gamma h-1}}{\Gamma(\gamma h)} \exp \left(\frac{1}{2} \gamma a h^{2}\right)+\frac{(\sigma x)^{\gamma h} C_{1}}{\sigma \Gamma(\gamma h+1)}+\frac{(\sigma x)^{\gamma h+1} C_{2}}{\sigma \Gamma(\gamma h+2)}+\cdots .
$$

So

$$
\frac{f_{Z_{1}}(x)}{x^{\gamma h-1}} \rightarrow \frac{(\sigma \beta)^{-\gamma h} \exp \left(\frac{1}{2} \gamma a h^{2}\right)}{\Gamma(\gamma h)} \text { as } x \rightarrow 0,
$$


and

$$
F_{Z_{1}}(x) \sim \frac{x^{\gamma h}(\sigma \beta)^{-\gamma h} \exp \left(\frac{1}{2} \gamma a h^{2}\right)}{\Gamma(\gamma h+1)} \text { as } x \rightarrow 0 .
$$

Thus, the distribution $F$ of $Z_{n}$ is regularly varying at 0 with exponent $\gamma h$.

From the definition of $k_{N}$ in (3.4) we have $1 / N=\int_{0}^{k_{N}} F_{Z_{1}}(\mathrm{~d} u)$. This equation, together with (5.3), gives

$$
k_{N}^{-1} \sim(\sigma \beta)^{-1}(\Gamma(\gamma h+1))^{-1 /(\gamma h)} \exp \left(\frac{1}{2} a h\right) N^{1 /(\gamma h)} \quad \text { as } N \rightarrow \infty .
$$

In order to calculate $c_{\gamma h}$ we need to find $\mathrm{E}\left[Y_{n}^{(h)}\right]^{\gamma h}$, where $Y_{n}^{(h)}=\sum_{j=0}^{\infty} \phi^{j} Z_{n-j}$. The Laplace transform of $Y_{n}^{(h)}$ is

$$
\tilde{f}_{Y_{n}^{(h)}}(s)=\mathrm{E} \exp \left(-s Y_{n}^{(h)}\right)=\prod_{j=0}^{\infty} \mathrm{E} \exp \left(-s \phi^{j} Z_{n-j}\right) .
$$

So

$$
\begin{aligned}
\log \tilde{f}_{Y_{n}^{(h)}}(s) & =\sum_{j=0}^{\infty} \log \tilde{f}_{Z_{1}}\left(s \phi^{j}\right) \\
& =\sum_{j=0}^{\infty} \log \tilde{f}_{W_{h}}\left(s \sigma \phi^{j}\right) \\
& =-\gamma \sum_{j=0}^{\infty} \int_{0}^{h} \log \left(1+\beta s \sigma \phi^{j} \mathrm{e}^{-a y}\right) \mathrm{d} y
\end{aligned}
$$

hence,

$$
\begin{aligned}
\tilde{f}_{Y_{n}^{(h)}}(s) & =\exp \left(\frac{\gamma}{a} \sum_{j=0}^{\infty}\left(\operatorname{dilog}\left(1+\beta s \sigma \phi^{j}\right)-\operatorname{dilog}\left(1+\beta s \sigma \phi^{j} \mathrm{e}^{-a h}\right)\right)\right) \\
& =\exp \left(\frac{\gamma}{a} \operatorname{dilog}(1+\beta s \sigma)\right),
\end{aligned}
$$

where dilog is the dilogarithm function,

$$
\operatorname{dilog}(x)=\int_{1}^{x} \frac{\log (u)}{1-u} \mathrm{~d} u .
$$

Using Theorem 2.1 of Brockwell and Brown (1978), we find, for $\gamma h<1$, that

$$
\begin{aligned}
\mathrm{E}\left[Y_{n}^{(h)}\right]^{\gamma h} & =\frac{1}{\Gamma(1-\gamma h)} \int_{0}^{\infty} s^{-\gamma h}\left|D \tilde{f}_{Y_{n}^{(h)}}(s)\right| \mathrm{d} s \\
& =\frac{\gamma}{a \Gamma(1-\gamma h)} \int_{0}^{\infty} s^{-\gamma h-1} \exp \left(\frac{\gamma}{a} \operatorname{dilog}(1+\beta s \sigma)\right) \log (1+\beta s \sigma) \mathrm{d} s
\end{aligned}
$$

where $D f$ denotes the derivative of $f$. Then $c_{\gamma h}=\left(\mathrm{E}\left[Y_{n}^{(h)}\right]^{\gamma h}\right)^{1 /(\gamma h)}$ can be numerically evaluated from (5.5) for fixed $h$. Theorem 2.1 also covers the case in which $\gamma h \geq 1$, but our prime concern here is with small values of $h$. 
Theorem 5.1. For a sequence of observations $\left\{Y_{n}^{(h)}, n=0,1, \ldots, N\right\}$ from a gamma-driven CAR(1) process, we have $\hat{a}_{N}^{(h)} \rightarrow$ a a.s. and

$$
\lim _{N \rightarrow \infty} \mathrm{P}\left[(-h) \mathrm{e}^{-a h} k_{N}^{-1}\left(\hat{a}_{N}^{(h)}-a\right) c_{\alpha} \leq x\right]=G_{\alpha}(x),
$$

where $G_{\alpha}$ is given in (3.5), $\alpha=\gamma h, \hat{a}_{N}^{(h)}$ is defined in (3.6), $k_{N}^{-1}$ is given in (5.4), and $c_{\alpha}$ is evaluated through (5.5).

Proof. At the beginning of Section 3 we showed that $Y_{n}^{(h)}$ is a stationary discrete-time AR(1) with autoregression coefficient $\phi \in(0,1)$ and driven by i.i.d. noise $\left\{Z_{n}\right\}$. According to (5.3), the distribution function $F$ of $Z_{n}$ is regularly varying at 0 with exponent $\alpha=\gamma h$ and satisfies the condition $F(0)=0$. Since $0 \leq Z_{n} \leq \sigma\left(L(n h)-L((n-1) h), \int u^{\xi} F(d u)<\infty\right.$ for all $\xi>0$. By Corollary 2.4 of Davis and McCormick (1989), we have $\hat{\phi}_{N}^{(h)} \rightarrow \phi$ a.s., which implies that $\hat{a}_{N}^{(h)} \rightarrow a$ a.s. From the same corollary, we also conclude that

$$
\lim _{N \rightarrow \infty} \mathrm{P}\left[k_{N}^{-1}\left(\hat{\phi}_{N}^{(h)}-\phi\right) c_{\gamma h} \leq x\right]=G_{\gamma h}(x),
$$

where $\hat{\phi}_{N}^{(h)}$ and $k_{N}^{-1}$ are given in (3.3) and (5.4), respectively, and $c_{\gamma h}$ is evaluated through (5.5). Using a Taylor series expansion, from this result we find that

$$
\lim _{N \rightarrow \infty} \mathrm{P}\left[(-h) \mathrm{e}^{-a h} k_{N}^{-1}\left(\hat{a}_{N}^{(h)}-a\right) c_{\gamma h} \leq x\right]=G_{\gamma h}(x) .
$$

Theorem 5.1 gives the limiting distribution of $\phi_{N}^{(h)}$ for fixed $h$ as $N$ tends to $\infty$. It is of interest also to consider the behavior of the estimator as $h$ also goes to 0 . For any nonnegative random variable $Y$ with density function $f(u)$, we have

$$
\begin{aligned}
{\left[\mathrm{E} Y^{s}\right]^{1 / s} } & =\left(\int_{0}^{\infty} u^{s} f(u) \mathrm{d} u\right)^{1 / s} \\
& =\left(1+s \int_{0}^{\infty} u^{s-1} f(u) \mathrm{d} u\right)^{1 / s} \\
& \rightarrow \exp \left(\int_{0}^{\infty} u^{-1} f(u) \mathrm{d} u\right) \\
& =\exp \left(\mathrm{E} Y^{-1}\right) \quad \text { as } s \rightarrow 0
\end{aligned}
$$

as long as $\mathrm{E} Y^{-1}$ is finite. Applying this result to $Y_{n}^{(h)}$, recalling the stationarity of the sequence $\left\{Y_{n}^{(h)}\right\}$, and using Theorem 2.1 of Brockwell and Brown (1978), we obtain

$$
\begin{aligned}
\lim _{h \rightarrow 0} c_{\gamma h} & =\exp \left(\mathrm{E}\left(Y_{n}^{(h)}\right)^{-1}\right) \\
& =\exp \left(\int_{0}^{\infty} \tilde{f}_{Y_{n}^{(h)}}(s) \mathrm{d} s\right) \\
& =\exp \left(\int_{0}^{\infty} \exp \left(\frac{\gamma}{a} \operatorname{dilog}(1+\beta s \sigma)\right) \mathrm{d} s\right) .
\end{aligned}
$$

The behavior of $k_{N}^{-1}$, defined in (5.4), is more complicated. Using l'Hôpital's rule, we have

$$
\lim _{s \rightarrow 0}-\frac{\log \Gamma(s+1)}{s}=-\lim _{s \rightarrow 0} \frac{\Gamma^{\prime}(s+1)}{\Gamma(s+1)}=-\Gamma^{\prime}(1)=\gamma_{\mathrm{E}},
$$


where $\gamma_{\mathrm{E}}$ is the Euler-Mascheroni constant, with numerical value $0.5772 \ldots$ Hence, $\lim _{h \rightarrow 0}[\Gamma(\gamma h+1)]^{-1 /(\gamma h)}=\exp \left(\gamma_{\mathrm{E}}\right)$ and

$$
\lim _{h \rightarrow 0} \lim _{N \rightarrow \infty} N^{-1 /(\gamma h)} k_{N}^{-1}=(\sigma \beta)^{-1} \exp \left(\gamma_{\mathrm{E}}\right)
$$

When $h$ is small, $k_{N}^{-1}$ and $c_{\gamma h}$ can be well approximated by (5.6) and (5.7). Since the rate of convergence in Theorem 5.1, as indicated by $k_{N}^{-1}$, increases as $h$ decreases and since the limiting distribution $G_{\gamma h}$ becomes degenerate as $h$ tends to 0 , this suggests the possibility of superconvergence of $\hat{a}_{N}^{(h)}$ to $a$ as $N$ tends to $\infty$ and $h$ tends to 0. In fact, in Section 6 we show that for any fixed $T>0, \hat{a}_{T / h}^{(h)} \rightarrow a$ a.s. as $h \rightarrow 0$.

Example 5.1. We now illustrate the estimation procedure with a simulated example. We simulated the gamma-driven CAR(1) process defined by

$$
\mathrm{d} Y(t)+0.6 Y(t) \mathrm{d} t=\sigma \mathrm{d} L(t), \quad t \in[0,5000],
$$

at times $0,0.001,0.002, \ldots, 5000$, using an Euler approximation. We set the parameter $\gamma$ of the standardized gamma process as 2 . We then sampled the process at intervals $h=0.01$, $h=0.1$, and $h=1$ by selecting every 10 th, 100 th and 1000 th value, respectively. We generated 100 such realizations of the process and applied the above estimation procedure to generate 100 independent estimates, for each $h$, of the parameters $a$ and $\sigma$. The sample means and standard deviations of these estimators are shown in Table 1, which illustrates the remarkable accuracy of the estimators.

To estimate the parameter $\gamma$ of the driving standardized gamma process, we used the following procedure. For each $h$ and each realization, we used the estimated CAR(1) parameters in (4.2) to generate the estimated increments $\Delta L_{n}^{(h)}, n=1, \ldots, 5000 / h$. We then added these in blocks of length $1 / h$ to obtain 5000 independent estimated increments of $L$ in one time unit. The histogram of the increments for one realization with $h=0.01$ is shown, together with the true probability density of $L(1)$, in Figure 1 . Even if we did not know that the background driving Lévy process is a gamma process, the histogram strongly suggests that this is the case. For each $h$ and for each realization of the process, we then used the sample mean $\hat{\gamma}$ of the estimated increments per unit time to estimate the parameter $\gamma$ of the driving standardized

TABLE 1: Estimated parameters based on 100 replicates on $[0,5000]$ of the gammadriven CAR(1) process (5.8) with $\gamma=2$, observed at times $n h, n=0, \ldots,\lfloor T / h\rfloor$.

\begin{tabular}{lccc}
\hline \multirow{2}{*}{$h$} & Parameter & \multicolumn{2}{c}{ Gamma increments } \\
\cline { 3 - 4 } & & $\begin{array}{c}\text { Sample mean } \\
\text { of estimators }\end{array}$ & $\begin{array}{c}\text { Sample standard } \\
\text { deviation of estimators }\end{array}$ \\
\hline \multirow{2}{*}{1} & $a$ & 0.59269 & 0.00381 \\
& $\sigma$ & 0.99796 & 0.01587 \\
\hline \multirow{2}{*}{0.1} & $a$ & 0.59999 & 0.00000 \\
& $\sigma$ & 1.00011 & 0.01281 \\
\hline \multirow{2}{*}{0.01} & $a$ & 0.60000 & 0.00000 \\
& $\sigma$ & 0.99990 & 0.01175 \\
\hline
\end{tabular}




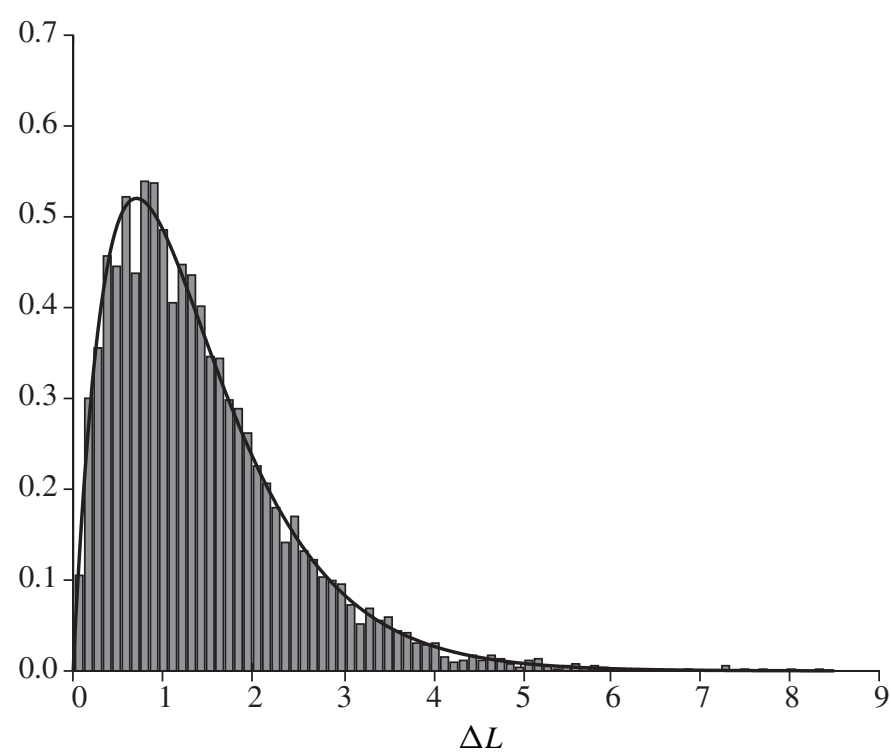

FIGURE 1: The probability density of the increments per unit time of the standardized Lévy process with $\gamma=2$ and the histogram of the estimated increments from a realization of the CAR(1) process (5.8), obtained by computing $\Delta L_{n}^{(.01)}, n=1, \ldots, 500000$, from (4.2) and adding successive values in blocks of 100 to give estimated increments per unit time.

TABLE 2: Estimated parameter of the standardized driving Lévy process.

\begin{tabular}{lccc}
\hline$h$ & Parameter & $\begin{array}{c}\text { Sample mean } \\
\text { of estimators }\end{array}$ & $\begin{array}{c}\text { Sample standard } \\
\text { deviation of estimators }\end{array}$ \\
\hline 1 & $\gamma$ & 1.99598 & 0.05416 \\
0.1 & $\gamma$ & 2.00529 & 0.03226 \\
0.01 & $\gamma$ & 2.00547 & 0.02762 \\
\hline
\end{tabular}

Lévy process, giving a set of 100 independent estimates of $\gamma$ for each $h$. The sample means and standard deviations of these estimators are shown in Table 2.

\section{Estimation for the continuously observed process}

It is interesting to note that, from a continuously observed realization on $[0, T]$ of a CAR(1) process driven by a nondecreasing Lévy process with drift $m=0$, the value of $a$ can be identified exactly with probability 1 . This contrasts strongly with the case of a Gaussian CAR(1) process. The result is a corollary of the following theorem.

Theorem 6.1. If the CAR(1) process $\{Y(t), t \geq 0\}$ defined by (2.1) is driven by a nondecreasing Lévy process $L$ with drift $m$ and Lévy measure $v$ then, for each fixed $t$,

$$
\frac{Y(t+h)-Y(t)}{h}+a Y(t) \rightarrow m \sigma \quad \text { a.s. as } h \downarrow 0 .
$$


Proof. From (2.6) we find that

$$
\begin{aligned}
Y(t+h)-Y(t)= & Y(0)\left(\mathrm{e}^{-a(t+h)}-\mathrm{e}^{-a t}\right)+\sigma(L(t+h)-L(t)) \\
& -a \sigma \int_{0}^{t} \mathrm{e}^{-a(t-u)}\left(\mathrm{e}^{-a h}-1\right) L(u) \mathrm{d} u-a \sigma \int_{t}^{t+h} \mathrm{e}^{-a(t+h-u)} L(u) \mathrm{d} u .
\end{aligned}
$$

Dividing each side by $h$, letting $h \downarrow 0$, and using the fact that $\lim _{h \downarrow 0}(L(t+h)-L(t)) / h=m$ (see Shtatland (1965)), we see that

$$
\frac{Y(t+h)-Y(t)}{h} \rightarrow m \sigma-a Y(0) \mathrm{e}^{-a t}+a^{2} \sigma \int_{0}^{t} \mathrm{e}^{-a(t-u)} L(u) \mathrm{d} u-a \sigma L(t)=m \sigma-a Y(t) .
$$

Corollary 6.1. If $m=0$ in Theorem 6.1 (this is the case if the point 0 belongs to the closure of the support of $L(1))$ then, for each fixed $t$, with probability 1 ,

$$
a=\lim _{h \downarrow 0} \frac{\log Y(t)-\log Y(t+h)}{h} .
$$

For each fixed $T>0$, a is also expressible, with probability 1, as

$$
a=\sup _{0 \leq s<t \leq T} \frac{\log Y(s)-\log Y(t)}{t-s} .
$$

Proof. By setting $L(t)=0$ for all $t$ in the defining equation (2.1) we obtain the inequality

$$
\log Y(s)-\log Y(t) \leq a(t-s)
$$

for all $s$ and $t$ such that $0 \leq s<t \leq T$, from which it follows that

$$
a \geq \sup _{0 \leq s<t \leq T} \frac{\log Y(s)-\log Y(t)}{t-s} .
$$

From Theorem 6.1 with $m=0$, we find that

$$
\frac{Y(t)-Y(t+h)}{h Y(t)} \rightarrow a \quad \text { as } h \downarrow 0 .
$$

From the inequalities in (6.3) and $1-x \leq-\log x$ for $0<x \leq 1$, we obtain the inequalities

$$
\frac{Y(t)-Y(t+h)}{h Y(t)} \leq \frac{\log Y(t)-\log Y(t+h)}{h} \leq a,
$$

and letting $h \downarrow 0$ gives (6.1). But this implies that

$$
a \leq \sup _{0 \leq s<t \leq T} \frac{\log Y(s)-\log Y(t)}{t-s},
$$

which, with (6.3), gives (6.2).

Remark 6.1. If observations are available only at times $\{n h: n=0,1,2, \ldots,\lfloor T / h\rfloor\}$, and if the driving Lévy process has zero drift, Corollary 6.1 suggests the following estimator:

$$
\hat{a}_{T}^{(h)}=\sup _{0 \leq n<\lfloor T / h\rfloor} \frac{\log Y(n h)-\log Y((n+1) h)}{h} .
$$


This estimator is precisely the same as the estimator (3.6). Its remarkable accuracy has already been illustrated in Table 1. The analogous estimator, based on closely but irregularly spaced observations at times $t_{1}, t_{2}, \ldots, t_{N}$ such that $0 \leq t_{1}<t_{2}<\cdots<t_{N} \leq T$, is

$$
\hat{a}_{T}=\sup _{n} \frac{\log Y\left(t_{n}\right)-\log Y\left(t_{n+1}\right)}{t_{n+1}-t_{n}} .
$$

By Corollary 6.1, both estimators converge almost surely to $a$ as the maximum spacing between successive observations converges to 0 .

\section{Conclusions}

Under the conditions specified in Section 3, we have examined the asymptotic properties of a highly efficient method, using observations at times $0, h, 2 h, \ldots, N h$, for estimating the parameters of a stationary Ornstein-Uhlenbeck process $\{Y(t)\}$ driven by a nondecreasing Lévy process $\{L(t)\}$. For small $h$, we used a discrete approximation to the exact integral representation of $L(t)$ in terms of $\{Y(s), s \leq t\}$ to estimate the increments of the driving Lévy process and, hence, to estimate the parameters of the Lévy process. Under the specified conditions, we obtained the asymptotic distribution of the estimator of the CAR(1) coefficient as $N$ tended to $\infty$ with $h$ fixed. The accuracy of the procedure was illustrated with a simulated example of a gamma-driven process. We also showed that the CAR(1) coefficient $a$ is determined almost surely by a continuously observed realization of $Y$ on any interval $[0, T]$. The expression for $a$ suggests an estimator based on discrete observations of $Y$ which, for uniformly spaced observations, is the same as the estimator developed in Section 3.

In Section 6 we found that if $L$ has zero drift, the estimator $\hat{a}_{T}^{(h)}$ based on $h$-spaced observations on $[0, T]$ is almost surely consistent as $h$ tended to 0 for any fixed $T>0$. The asymptotic distribution, as $T$ tended to $\infty$ with $h$ fixed, was computed explicitly in Section 5 for the case in which $L$ is a gamma process. The critical step was the establishment of the regular variation of the distribution function of $\int_{0}^{h} \mathrm{e}^{-a u} \mathrm{~d} L(u)$ at 0 . More generally, if $\mathrm{E}^{-s L(t)}=\mathrm{e}^{-t \Phi(s)}$, where $\Phi(s)=c \log (s)+\sum_{j=0}^{\infty} c_{j} s^{-j}$ for $|s|>R$, then the same argument as in Section 5 shows that the distribution of the integral is again regularly varying at 0 and that the exponent is $c h$. In fact, the regular variation (RV) condition depends only on the behavior of the Lévy measure $v$ near 0 . This can be shown as follows. For any given $\varepsilon>0$, the process $L(t)$ can be written as the sum of two independent Lévy processes

$$
L(t)=S(t)+B(t)
$$

where $S$ has Lévy measure $v((0, \varepsilon] \cap \cdot)$ and $B$ has the finite Lévy measure $v([\varepsilon, \infty) \cap \cdot)$. Provided that $x<\varepsilon \mathrm{e}^{-a h}$, the distribution function of $\int_{0}^{h} \mathrm{e}^{-a u} \mathrm{~d} L(u)$ can then be expressed as

$$
F(x)=\mathrm{P}\left(\int_{0}^{h} \mathrm{e}^{-a u} \mathrm{~d} L(u) \leq x\right)=\mathrm{P}\left(\int_{0}^{h} \mathrm{e}^{-a u} \mathrm{~d} S(u) \leq x\right) \mathrm{e}^{-h v((\varepsilon, \infty))} .
$$

This shows that $F(c x) / F(x)$ is independent of $v((\varepsilon, \infty) \cap \cdot)$ for $x<\varepsilon \mathrm{e}^{-a h}$. If, in particular, the Lévy density coincides with that of the gamma process $\left(\gamma x^{-1} \mathrm{e}^{-\beta x}\right)$ on some interval $(0, \varepsilon]$ then the RV condition is satisfied. This leads to the conjecture that a Lévy density of the form $\gamma x^{-1}(1+o(x))$ ensures satisfaction of the RV condition with exponent $\gamma h$.

If the Lévy density of $L$ has the form $\gamma x^{-1-\beta}$, with $\gamma>0$ and $0<\beta<1$, then $L(h)$ and $Z_{1}:=\sigma \int_{0}^{h} \mathrm{e}^{-a(h-t)} \mathrm{d} L(t)$ are both positive stable random variables with exponent $\beta$ and 
the $\mathrm{RV}$ condition is not satisfied. This is readily seen when $\beta=\frac{1}{2}$, in which case the density of $Z_{1}$ has the form $f(x)=\sqrt{c /(2 \pi)} x^{-1.5} \mathrm{e}^{-c / 2 x}$. (For the general case, there is no concise explicit form of the density function, but a series expansion can be found in Brockwell and Brown (1978).) The density $f(x)$ approaches 0 at a much faster rate as $x$ tends to 0 than in the gamma case, and since the success of our estimator depends upon the existence of an interval of length $h$ with a very small increment in $L$, it should be less efficient in this case than in the gamma case.

If, on the other hand, the Lévy density of $L$ is less than or equal to $\gamma x^{-1-\beta}$, with $\beta<0$, in some neighborhood of 0 , then $L$ has finite Lévy measure and the distributions of $L(h)$ and $Z_{1}$ both have positive mass at 0 . Hence, with probability $1, \min \left\{n>0: Y(n h)=\mathrm{e}^{-a h} Y((n-1) h)\right\}$ is finite for any fixed $h>0$, and the estimator $\hat{a}_{T}^{(h)}$ is equal to $a$ for sufficiently large $T$.

The generalization of the procedure to nonnegative Lévy-driven continuous-time ARMA processes is currently in progress.

\section{Acknowledgements}

We are indebted to the National Science Foundation for support of this work under Grants DMS-0308109, DMS 0743459, and DMS 0744058. P. J. Brockwell is grateful for the additional support of Deutsche Forschungsgemeinschaft, SFB 386, at Technische Universität München. We also gratefully acknowledge the valuable comments of an anonymous referee and an editor, particularly with regard to the RV condition.

\section{References}

Barndorff-Nielsen, O. E. ANd ShePhard, N. (2001). Non-Gaussian Ornstein-Uhlenbeck-based models and some of their uses in financial economics. J. R. Statist. Soc. Ser. B 63, 167-241.

Brockwell, P. J. AND Brown, B. M. (1978). Expansions for the positive stable laws. Z. Wahrscheinlichkeitst. 45, 213-224.

BrockWell, P. J. AND Marquardt, T. (2005). Fractionally integrated continuous-time ARMA processes. Statistica Sinica 15, 477-494.

DAvis, R. AND McCormick, W. (1989). Estimation for first-order autoregressive processes with positive and bounded innovations. Stoch. Process. Appl. 31, 273-250.

Doetsch, G. (1974). Introduction to the Theory and Application of the Laplace Transformation. Springer, New York.

Jongbloed, G., van der Meulen, F. H. And van Der VAart, A. W. (2005). Nonparametric inference for Lévy-driven Ornstein-Uhlenbeck processes. Bernoulli 11, 759-791.

Phillips, A. W. (1959). The estimation of parameters in systems of stochastic differential equations. Biometrika 46, 67-76.

Protter, P. E. (2004). Stochastic Integration and Differential Equations, 2nd edn. Springer, New York.

Shtatland, E.S. (1965). On local properties of processes with independent increments. Theory Prob. Appl. 10, 317-322. 